\title{
Microsurgical salvage of complex dorsal shearing injuries of the hand and wrist
}

\author{
Praveen G. Murthy ${ }^{1,2, *}$, Adam B. Strohl'1,2,3,* \\ 1Philadelphia Hand to Shoulder Center, Philadelphia, PA 19107, USA. \\ ${ }^{2}$ Department of Orthopaedics, Thomas Jefferson University Hospital, Philadelphia, PA 19107, USA. \\ ${ }^{3}$ Department of General Surgery - Plastic Surgery, Thomas Jefferson University Hospital, Philadelphia, PA 19107, USA. \\ *Both authors contributed equally to the article.
}

Correspondence to: Dr. Adam B. Strohl, Philadelphia Hand to Shoulder Center, 834 Chestnut Street Suite G-114, Philadelphia, PA 19107, USA.E-mail: abstrohl@handcenters.com

How to cite this article: Murthy PG, Strohl AB. Microsurgical salvage of complex dorsal shearing injuries of the hand and wrist. Plast Aesthet Res 2020;7:64. http://dx.doi.org/10.20517/2347-9264.2020.72

Received: 12 Apr 2020 First Decision: 9 Sep 2020 Revised: 24 Sep 2020 Accepted: 23 Oct 2020 Published: 13 Nov 2020

Academic Editor: Alessandro Thione Copy Editor: Cai-Hong Wang Production Editor: Jing Yu

\begin{abstract}
Dorsal shearing injuries of the hand and wrist can be seen with high-energy motor vehicle accidents and present challenging problems to the reconstructive surgeon. At the time of initial injury, care must be taken to adequately debride the mangled extremity and extensive wounds. Following debridement, a series of decisions must be made regarding bony stabilization, extensor tendon reconstruction, and soft tissue coverage. These reconstructions often require staged procedures, and appropriate planning is warranted from the start. Reasonable function of the hand can be expected from the patient following such injuries.
\end{abstract}

Keywords: Dorsal shear, hand, wrist, trauma, extensor, free flap, mangling

\section{INTRODUCTION}

Mangling injuries of the upper extremity are devastating injuries involving a combination of multiple tissue layers and structures that are critical for hand function. Among these, traumatic dorsal shearing injuries of the hand and wrist pose unique challenges with regards to soft tissue and bony reconstruction ${ }^{[1,2]}$.

Dorsal shearing injuries generally involve the skin and soft tissue envelope covering the dorsal hand and wrist, the extensor tendons, and often the bony structures that comprise the many complex articulations of

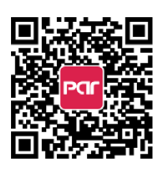


the wrist and hand. Such injuries can lead to significant problems with bony stability and extensor tendon function.

There is limited information in the current literature to guide management of complex dorsal hand and wrist injuries. In this article, we discuss the challenges associated with treating these injuries, provide an algorithm for management, and review a series of representative cases presenting to our tertiary referral center.

\section{INITIAL EVALUATION}

Dorsal shearing hand injuries are often associated with high energy trauma. It is imperative first to evaluate the patient using standard advanced trauma life support (ATLS) protocol with our trauma colleagues, including complete primary and secondary surveys, prior to addressing the more obvious injury to the hand. If and when the patient is deemed stable from a systemic perspective, focused evaluation of the hand is appropriate.

A thorough history is obtained if possible, including the timing of injury; where the injury occurred, with particular attention to the degree of contamination of the wound; and the mechanism of injury. Such details provide important information regarding the zone of injury and the quality of the underlying soft tissues for subsequent repair or reconstruction. Gross contamination can lead to infection and doom any achievements in reconstruction. Associated crush forces and thermal burns can be expected to cause secondary injuries in the time period following injury, thereby expanding the zone of obvious injury.

While detailed examination of the injured extremity in the emergency department is often difficult, a preliminary evaluation should focus primarily on two questions: (1) Are the fingers vascularized? (2) Are the fingers sensate?

Dorsal shearing injuries, by definition, spare the critical vascular and nervous structures that travel on the volar aspect of the hand and wrist. Unlike with many mangling injuries to the hand, microsurgical repair of vessels and nerves is generally not required to restore functionality in these cases. Once the patient is deemed to have a dorsal shearing injury with a vascularized and sensate hand, the goals of treatment become simplified. Formal assessment of the degree injury to each of the dorsal tissue layers may be performed in the operating room.

Preoperative radiographic examination of the forearm, wrist, and hand is essential to determine the nature of the skeletal injury and prompt fixation planning. The addition of embedded hardware with likely exposure within the wound will prompt additional coverage concerns. Any open fractures identified by exam and/or radiographs should be treated with prompt administration of broad-spectrum intravenous antibiotics.

The goals of management of complex dorsal hand injuries are as follows: (1) debridement; (2) bony stabilization; (3) stable soft tissue coverage; and (4) extensor tendon reconstruction.

\section{DEBRIDEMENT}

Thorough, careful excisional debridement is perhaps the most important step in ensuring a favorable result ${ }^{[1]}$. Wound infection after mangling injuries can be devastating and can negate any benefit gained by surgical reconstruction. As such, any reconstructive procedures should be delayed until the wound bed is clean and free of any devitalized tissue. If the patient is hemodynamically stable, debridement can be done without tourniquet to allow for visualizing of bleeding surfaces, signifying viability. No attempt should be 


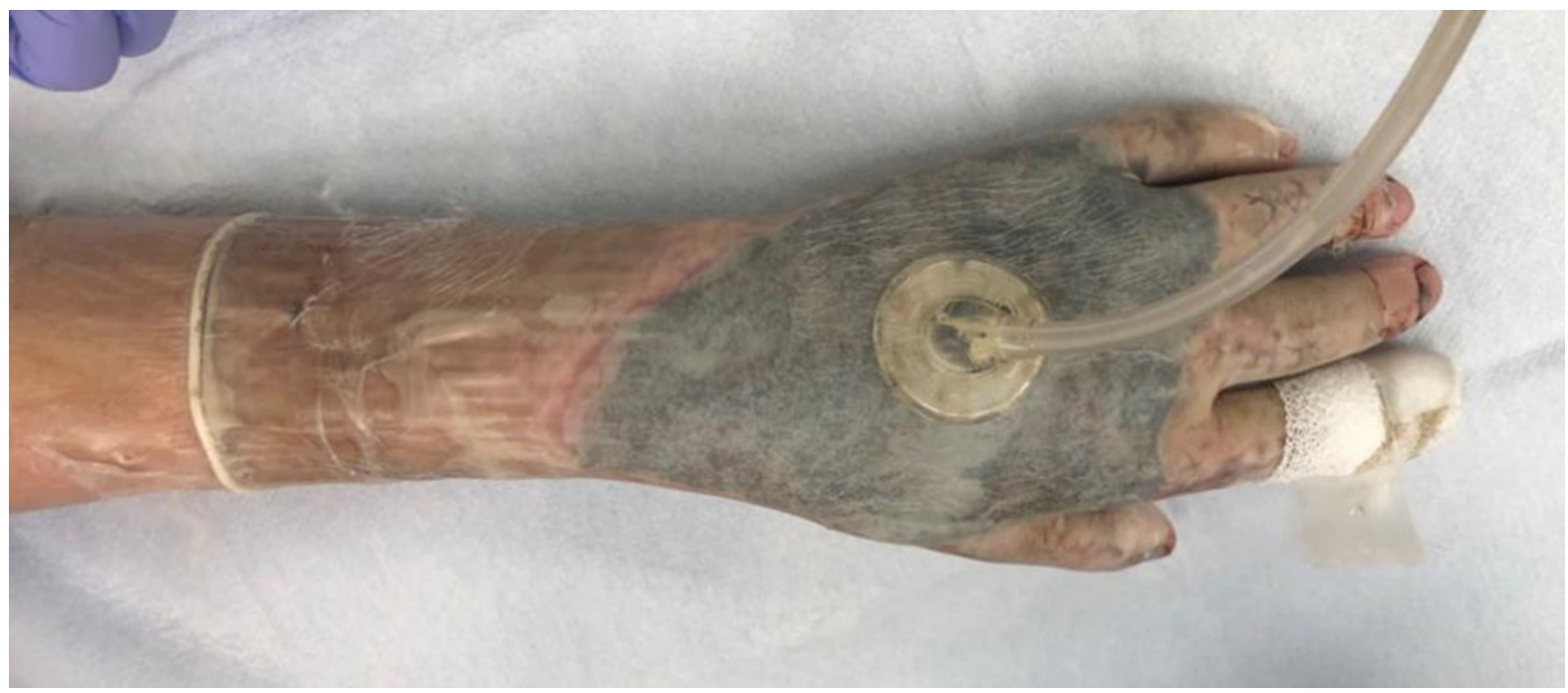

Figure 1. Negative pressure wound therapy can be used despite difficult-to-seal webspaces with the use of a sterile surgical glove

made at this step to minimize the wound dimensions and/or depth. This often requires multiple trips to the operating room for serial debridement, particularly in the case of farming injuries or motor vehicle accidents with significant environmental debris, as the extent of contamination and devitalization may not be immediately evident at the index procedure.

If serial debridement is deemed necessary, provisional skeletal stabilization can be achieved via external fixation. Negative pressure wound therapy is also beneficial when definitive wound coverage must be delayed. We use a "glove technique" [Figure 1] in order to facilitate vacuum-assisted closure in the setting of a complex and irregular soft tissue injury of the hand. A sterile, surgical glove can be placed over the sponge dressing and then sealed at the wrist level before applying the negative pressure via hose. This technique is ideal for "sealing" the webspaces from subsequent leak in the interoperative periods.

\section{BONY STABILIZATION}

Once the wound bed is deemed appropriate to proceed with reconstruction, the first goal of management is skeletal stabilization. Internal fixation of fractures may be pursued where able; however, dorsal shearing injuries of the wrist and hand can be associated with significant bone loss and comminuted articular fracture dislocations that are not amenable to anatomic reduction and stable internal fixation.

External fixation or spanning bridge plate technique may be used for definitive stabilization when applicable, as in cases with high energy distal radius fractures or radiocarpal dislocations with intact carpal bones. When using an external fixator, hardware should be placed as far from the zone of injury as possible without compromising the stability of the construct. One should be mindful of causing additional injury to nerves and to vessels that may allow for later anastomosis with free tissue transfer. Percutaneous wire fixation can be used but may lack the necessary stability in the setting of significant comminution and/ or bone loss. In cases with significant bone loss and/or complex articular fracture dislocations involving multiple articulations in the wrist and hand, acute total wrist arthrodesis may be the most reliable solution.

If the metacarpophalangeal joints have been destroyed, reconstruction with arthroplasties can be considered. A cement spacer can be used to maintain the joint space if immediate arthroplasty is not performed in the acute setting. The metacarpophalangeal joint has been successfully reconstructed with the transfer of a vascularized toe joint ${ }^{[3,4]}$. Reconstruction of multiple joints may limit this option in such dorsal shearing injuries. 


\section{SOFT TISSUE COVERAGE}

In the context of complex dorsal shearing injuries with extensor tendon disruption, the restoration of a gliding bed for reconstructed extensor tendons is critical. Vascularized soft tissue coverage is imperative in these cases, as non-vascularized skin grafts and skin substitutes will invariably result in extensor tendon adhesions. Several options for vascularized soft tissue coverage of the dorsal hand have been described in the literature, including pedicled and free fasciocutaneous flaps ${ }^{[5-9]}$, fascial flaps ${ }^{[10,11]}$, and muscle flaps ${ }^{[12-15]}$.

Pedicled fasciocutaneous flaps, including distally-based reverse $\operatorname{radial}^{[6]}$ and ulnar artery ${ }^{[7]}$ flaps, are technically less demanding than free flaps, but they sacrifice a major supply of blood flow to an already comprised area, and may be insufficient for coverage of larger defects. The reverse posterior interosseous artery flap ${ }^{[8]}$ is similarly limited in its dimensions. Additionally, perforator-based fasciocutaneous flaps such as the radial artery perforator flap negate the sacrifice of a major artery to the hand but have a higher complication rate and are limited to $8 \mathrm{~cm} \times 10 \mathrm{~cm}$ dimension ${ }^{[16,17]}$.

Several free fasciocutaneous flaps have been described with a common favorite being the free anterolateral thigh flap ${ }^{[9]}$. This is an ideal option for coverage of large dorsal hand defects. In particular, we recommend utilizing a fasciocutaneous flap when a secondary operation requiring flap elevation is likely, as in the case of delayed bone, joint, and/or tendon reconstruction.

Various muscle flaps are also well-suited for dorsal hand coverage, including the latissimus dorsi, rectus abdominus, and serratus anterior ${ }^{[12-15]}$. A muscle flap carries the advantage of more robust vascularity, but it can create a less favorable environment for reoperation. In our experience, healed muscle flaps can be more difficult to elevate than fasciocutaneous flaps in this area. As such, a muscle flap is more ideal when secondary surgery is not anticipated, or significant dead space is created by bone loss. Staged tendon reconstruction with silastic tendon implants can be performed without flap elevation and is not a contraindication to utilizing a muscle flap.

More recently, several authors have described single-stage reconstruction of dorsal hand defects with composite cutaneotendinous flaps, including the dorsalis pedis cutaneotendinous flap ${ }^{[18]}$ and the composite anterolateral thigh flap taken with fascia lata ${ }^{[19-21]}$. Composite flaps including bone have also been described, such as the free serratus anterior fascial flap with vascularized scapula for combined soft tissue and bony defects of the dorsal hand ${ }^{[22]}$. The primary disadvantage of composite flaps, particularly the cutaneotendinous dorsalis pedis flap or anterolateral thigh fascia lata flap, is the chance of considerable donor site morbidity. Additionally, a considerable amount of planning is needed to ensure correct orientation, placement, and size of the chimeric flap. Reconstructive surgeons around the world have also had success with "successive flap" or "daisy-chain" reconstruction, utilizing multiple free vascularized flaps connected via flow-through anastomosis at the terminal end ${ }^{[23]}$. In this fashion, dorsal soft tissue coverage could be supplied by previously described flaps and could then be connected to a free vascularized toe joint.

\section{EXTENSOR TENDON RECONSTRUCTION}

The primary challenge in extensor tendon reconstruction in the context of complex dorsal shearing injuries is the loss of a gliding plane for tendon excursion. One critical element of restoring a gliding plane involves the introduction of vascularized soft tissue coverage, as detailed in the previous section. The second element involves restoration of a tendon pseudosheath. While primary extensor tendon reconstruction in dorsal hand injuries has been described, the results have been mixed ${ }^{[2,25]}$. We prefer staged extensor tendon reconstruction using silicone tendon implants (often referred to somewhat inaccurately as "rods"), in order to ensure that tendon grafting is ultimately performed in a healthy wound bed that will allow for optimal tendon gliding. 
Staged tendon reconstruction using a silicone implant was first described by Hunter in $1965^{[26]}$. His technique is well-known and commonly used for staged flexor tendon reconstruction ${ }^{[27]}$, although, in fact, the first case demonstrating the utility of a silastic implant for tendon surgery was performed in 1960 and involved reconstruction of the extensor tendon to the index finger ${ }^{[26]}$.

The surgical technique is relatively straightforward ${ }^{[27]}$. In the first stage, silicone tendon implants are placed beneath the flap and secured to the extensor tendon stumps distally using 4-0 Prolene suture, outside of the zone of injury. To reconstruct four tendons, two such implants can be passed and split into a Y configuration distally to attach to two extensor tendons each or four separate units can be used. The proximal ends of the implants are trimmed at the appropriate level and left freestanding in a subcutaneous pocket in the distal forearm.

In the second stage, tendon grafts are harvested, secured to the proximal ends of the silicone implants, and then passed from proximal to distal through the new pseudosheath by pulling the implants distally. To reconstruct four tendons, two tendon grafts are harvested, passed, and similarly split into a Y configuration distally. The distal junctures are performed via Pulvertaft weave using non-absorbable suture. Proximally, the tendon grafts may be powered either by the extensor digitorum communis if sufficient proximal tendon remains, or by the flexor carpi radialis via tendon transfer. The proximal tendon juncture is performed similarly via Pulvertaft weave. The overlying flap is then sutured back into place, and early short-arc extensor tendon transfer/reconstruction protocols are begun postoperatively.

If staged reconstruction is not chosen, one could consider immediate tendon transfer for restoration of extensor function. Free tendon donor grafts can be used as immediate interposition grafts to restore anatomical continuity or in conjunction with tendon transfers such as flexor carpi ulnaris or flexor carpi radialis, especially if the wrist has been fused. A side-to-side tenodesis of injured extensor slips to adjacent non-injured slips can be effective to restore finger extension. A potential pitfall of this technique is adhesions of the tendon grafts or transfers beneath the healing flap. Finally, one may consider tenodesis of the distal extensor tendons such as extensor pollicis longus (EPL) or extensor digitorum communis to the metacarpal or radius for passive opening of the hand, as described in the spinal cord and brachial plexus populations.

\section{Postoperative rehabilitation}

Preserving motion throughout the phases is paramount to ultimate success of the reconstruction. Joints that are not directly injured cannot be neglected during the perioperative periods or else additional therapy, and even surgery, may be indicated to address stiffness. We advocate passive range of motion of uninvolved joints until active modalities can be resumed or restored by reconstruction. This is particularly important in the week or so immediately after flap reconstruction.

Once the soft tissue reconstruction is stable, we begin formal hand therapy, typically as early as Week 2, including such passive range of motion and appropriate splinting. Digits that are not directly involved can begin active range of motion and joint mobilization therapies. Joint arthroplasties are typically splinted in extension for four weeks while the proximal interphalangeal and distal interphalangeal joints can receive passive and/or active range of motion exercises as well as tendon glides, as indicated by the extensor status. Therapists can also focus on edema control and scar management throughout maturation of the reconstruction. For those patients requiring second stage extensor reconstruction, we perform this no sooner than eight weeks after the first stage with stable equilibrium of the soft tissue envelope. 


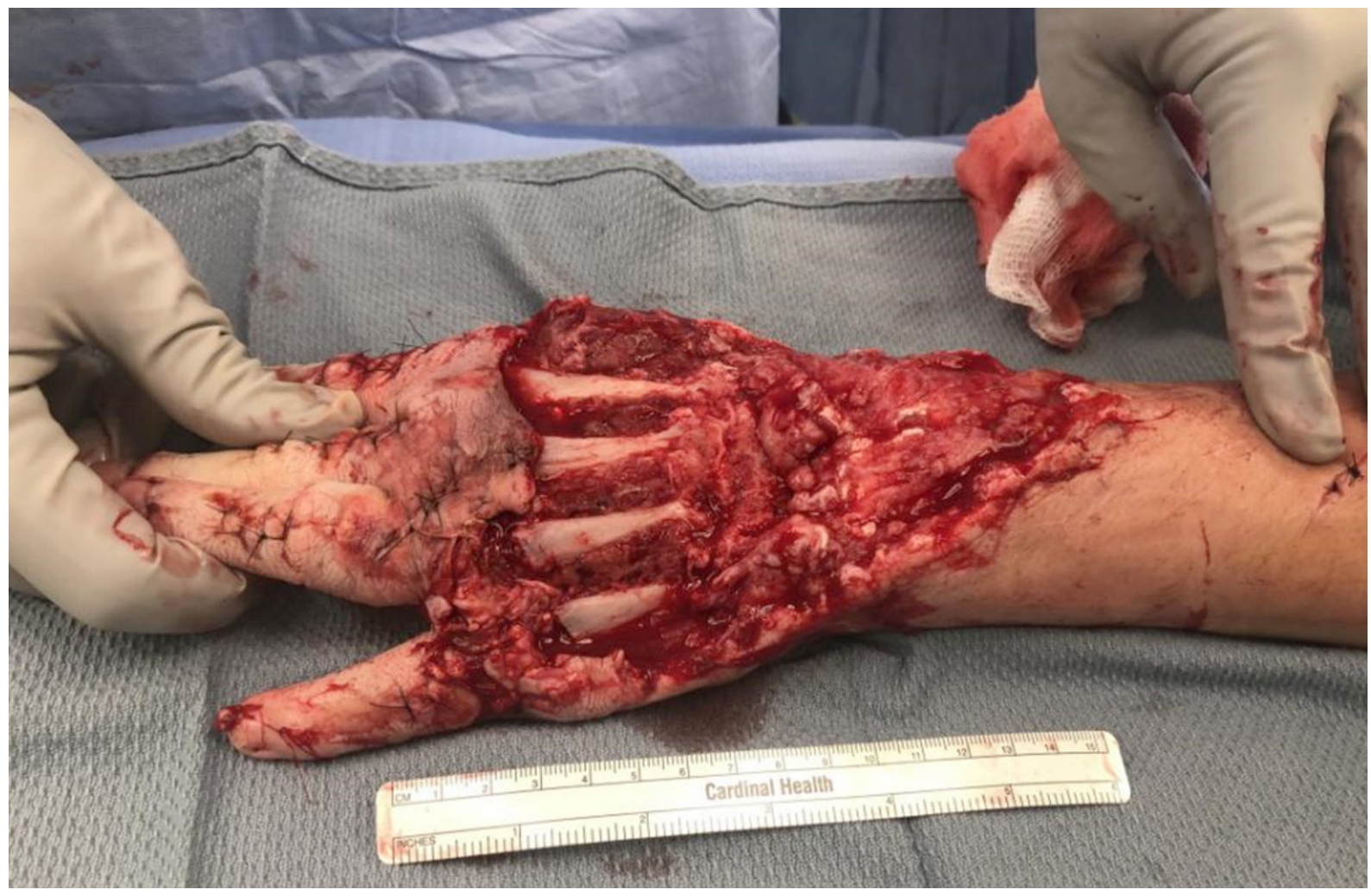

Figure 2. Following debridement, extensive destruction of the soft tissue envelope, extensor tendons (extensor digitorum communis, extensor indicis, and extensor digiti minimi), and metacarpophalangeal joints is noted

\section{CASE EXAMPLES}

\section{Case 1}

A 19-year-old male was transferred to our trauma center after a motor vehicle accident. He was an unrestrained driver and was ejected from the car, sustaining multiple injuries including a dorsal shearing injury of the left hand. Focused examination of the extremity revealed that the fingers were well-perfused and sensate. He was able to flex but unable to extend the fingers except the thumb.

He was taken to the operating room for exploration and debridement [Figure 2]. He was found to have significant skin and soft tissue loss; segmental loss of the extensor tendons to all fingers and laceration of the wrist extensors; open arthrotomies of the midcarpal and carpometacarpal joints; open fracture dislocations of the metacarpophalangeal (MP) joints of the index through small fingers; open arthrotomy long finger proximal interphalangeal joint; and dorsal radial sensory nerve loss.

At the index procedure, he underwent thorough excisional debridement and vacuum-assisted closure. He returned to the operating room every 2-3 days for seven subsequent debridements. After this time, the wound bed was deemed appropriate for definitive soft tissue coverage. He underwent free anterolateral thigh flap from the right thigh to the left hand and wrist, along with placement of antibiotic cement spacers in the MP joints of the index through small fingers [Figure 3]. Fasciocutaneous flap was used in this case to allow ease of lifting the flap for the planned, secondary procedures.

Four months later, he underwent silastic MP arthroplasty of the index through small fingers and first stage extensor tendon reconstruction of index through long fingers with placement of silastic tendon implants (Wright Medical Technology, Memphis, TN), as described above [Figure 4]. Three months after this, he 


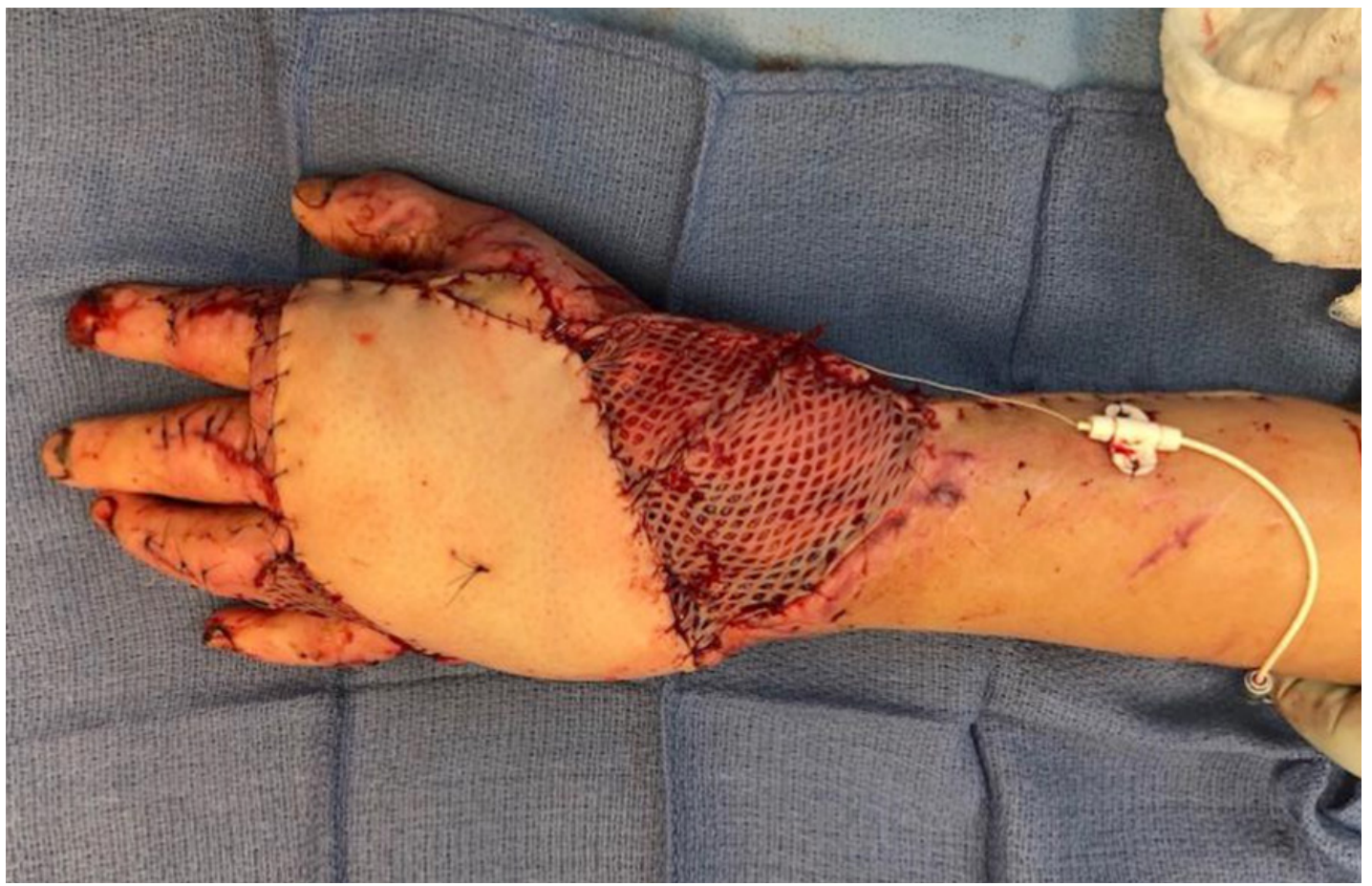

Figure 3. Free antero-lateral thigh flap with split thickness skin graft as well as cement spacers to the metacarpophalangeal joints of index through small fingers. Seen in the photograph is the implantable Synovis Flow Coupler (Baxter, Birmingham, AL) device for postoperative monitoring purposes

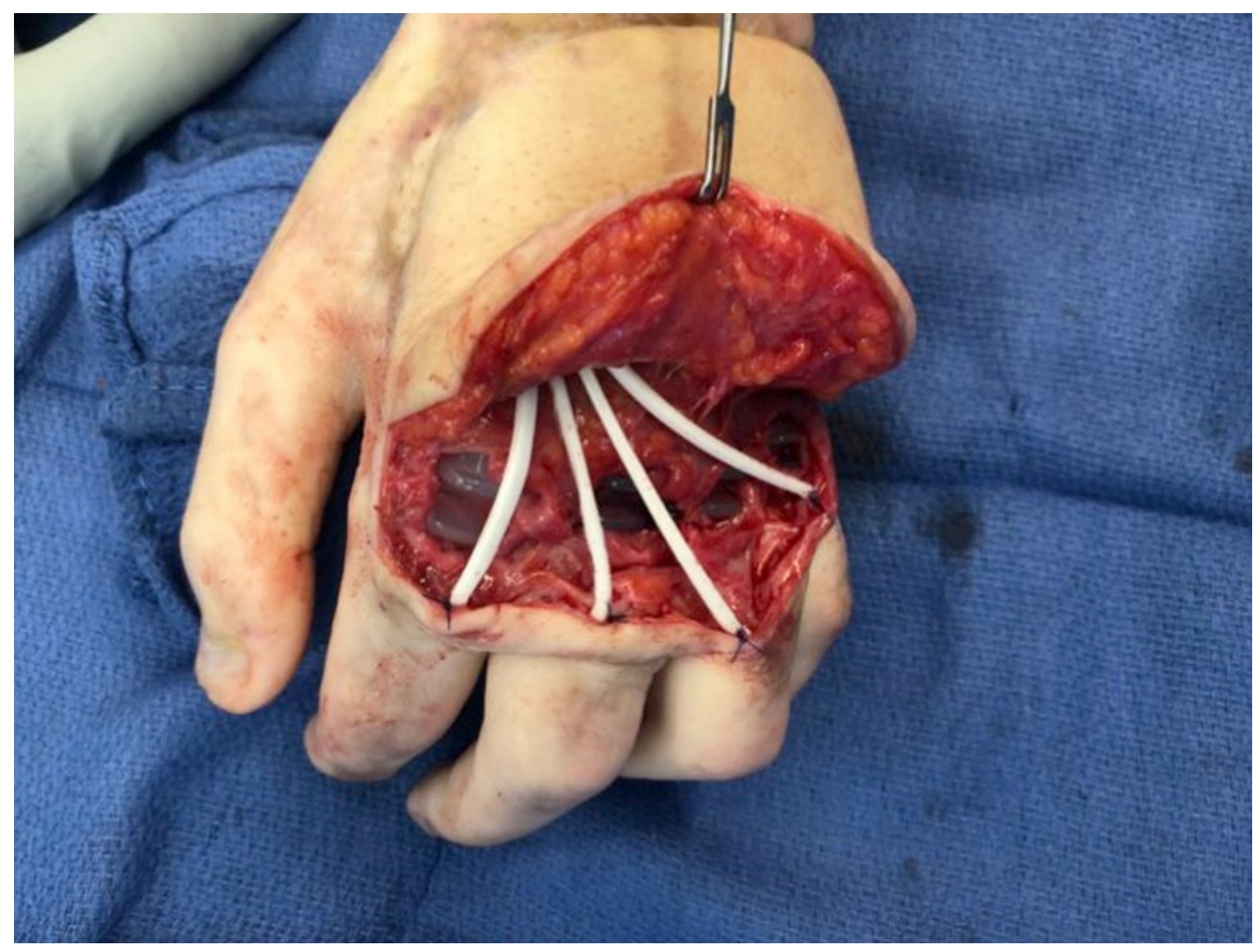

Figure 4. Staged extensor tendon reconstruction with silastic tendon implant placement and silastic arthroplasties (Integra LifeSciences, Princeton, NJ) of the index through small finger metacarpophalangeal joints 

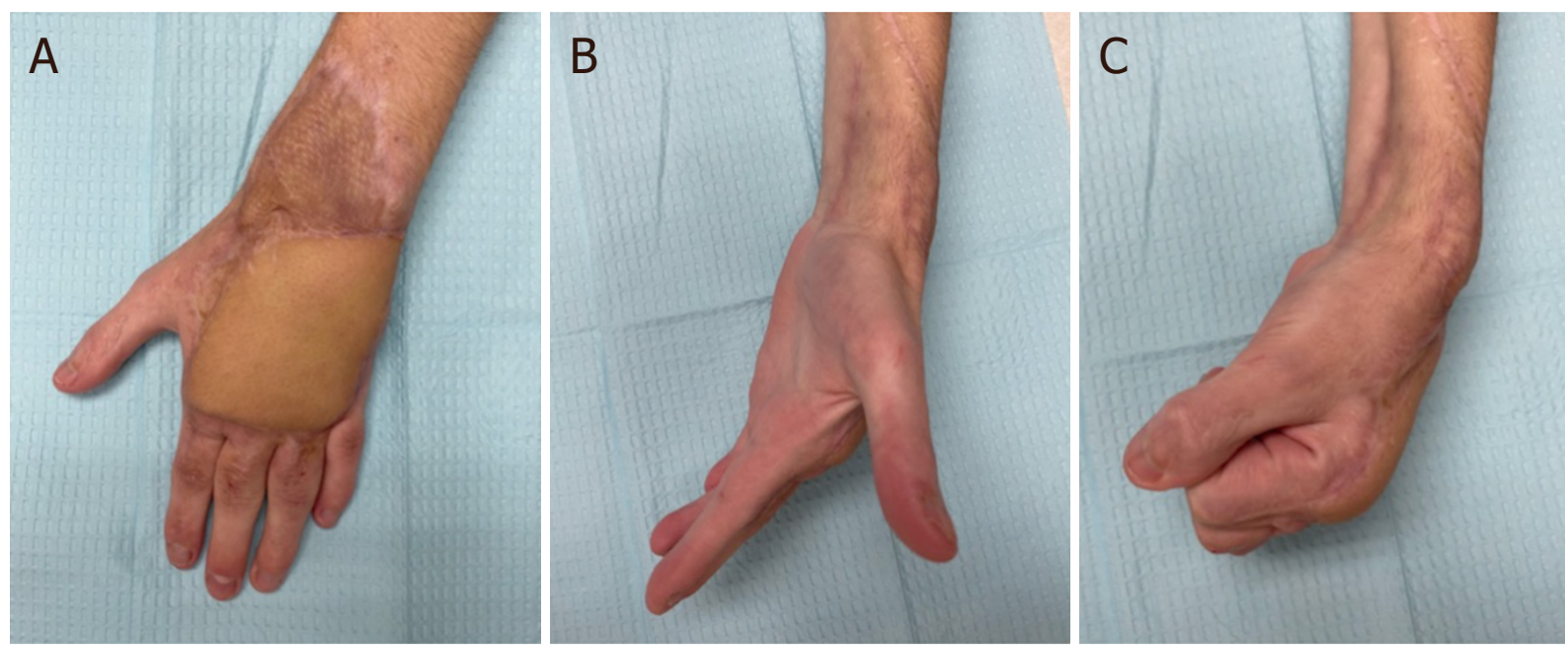

Figure 5. Appearance of reconstructed hand following suction lipectomy of the ALT flap for contour improvement and regular sessions of hand therapy $(A-C)$. Patient has pain-free, functional arc of flexion and extension of the digits with aesthetically pleasing result

underwent second stage extensor tendon reconstruction. Bilateral palmaris longus tendon grafts were harvested. These were passed from proximal to distal into the pseudosheath using the silicone implants, then split into a Y configuration distally, and sutured to each of the extensor tendons distally via Pulvertaft weave.

Finally, six months later, he underwent suction lipectomy for re-contouring of the left anterolateral thigh flap. At latest follow up, the flap was healthy, and he had reasonable function of the hand and was able to grip [Figure 5]. He had active extension of the digits enough to allow for typing and video-gaming. He could flex his fingers to touch the palm but not the distal palmar crease, and he was able to grasp objects. He returned to driving and working as a store clerk.

\section{Case 2}

A 47-year-old male was transferred to our trauma center after a rollover motor vehicle accident. He sustained an isolated dorsal shearing injury of the left wrist and hand with heavy, gross contamination of road and field debris. Focused examination of the extremity revealed that the fingers were well-perfused.

He was taken to the operating room for exploration and debridement [Figure 6]. He was found to have significant skin and soft tissue loss; segmental loss of the extensor tendons to all fingers; and open coronal plane fractures of the distal radius, distal ulna, scaphoid, lunate, capitate, hamate, and long and ring finger metacarpals.

At the index procedure, he underwent thorough excisional debridement, placement of antibiotic cement beads, and vacuum-assisted closure. Skeletal stabilization was achieved via external fixation, spanning from the radial diaphysis to the index metacarpal. He was brought back to the OR every 2-3 days for a total of three subsequent debridements. After this time, the wound was felt to be appropriate to proceed with definitive bony stabilization and soft tissue reconstruction.

For bony stabilization, he underwent total wrist arthrodesis using a Synthes 3.5-mm metaphyseal locking compression plate (DePuy Synthes, Raynham, MA) spanning from the distal radius to the third metacarpal, with additional stabilization using a Synthes $2.7-\mathrm{mm}$ reconstruction plate from the distal radius to the fourth metacarpal [Figure 7] For soft tissue reconstruction, he underwent free latissimus dorsi muscle flap 


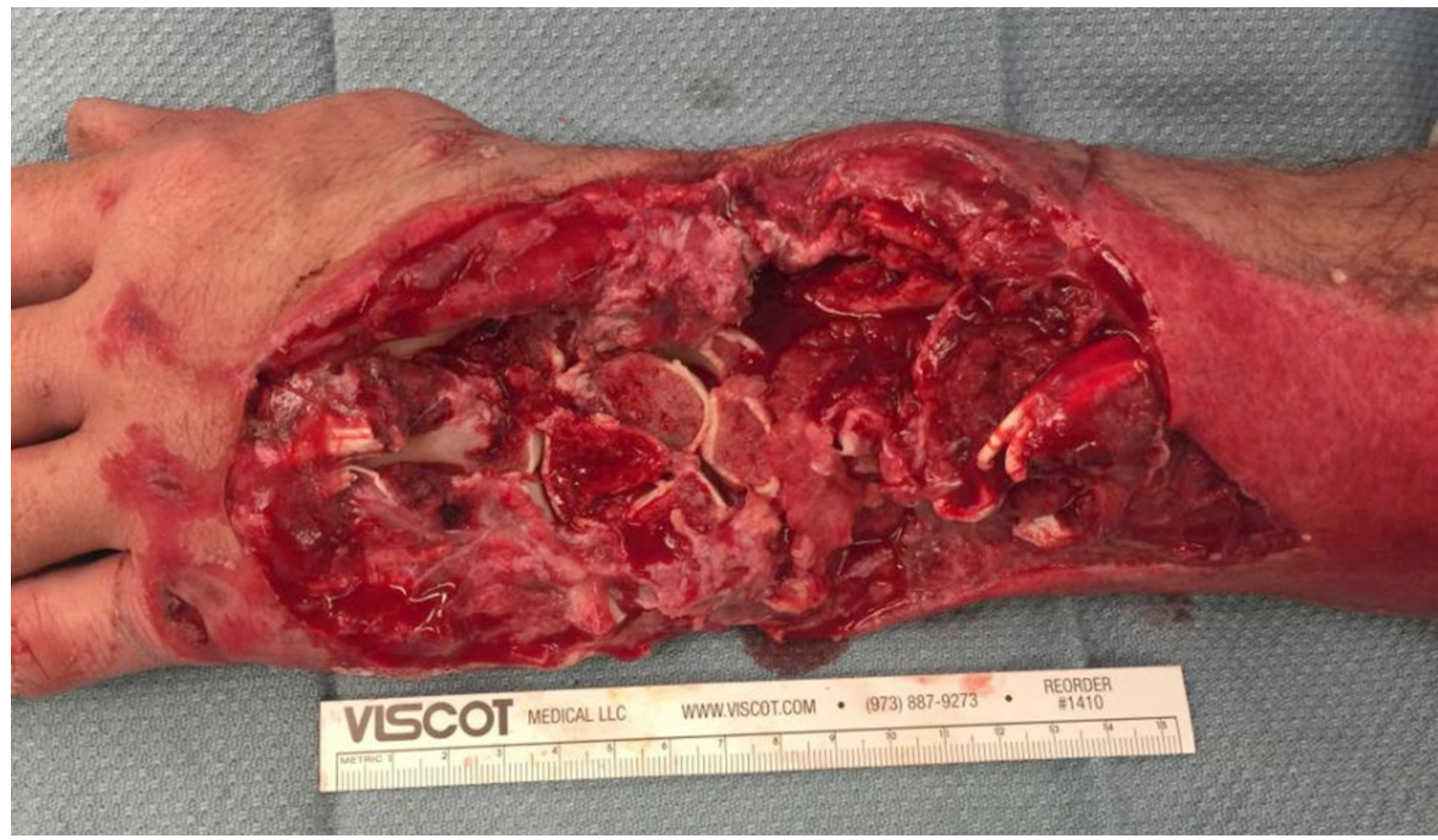

Figure 6. Following thorough extensive debridement of non-vitalized tissues and bone resulting in large wound with exposed bony defects, carpal instability, and extensor tendon loss
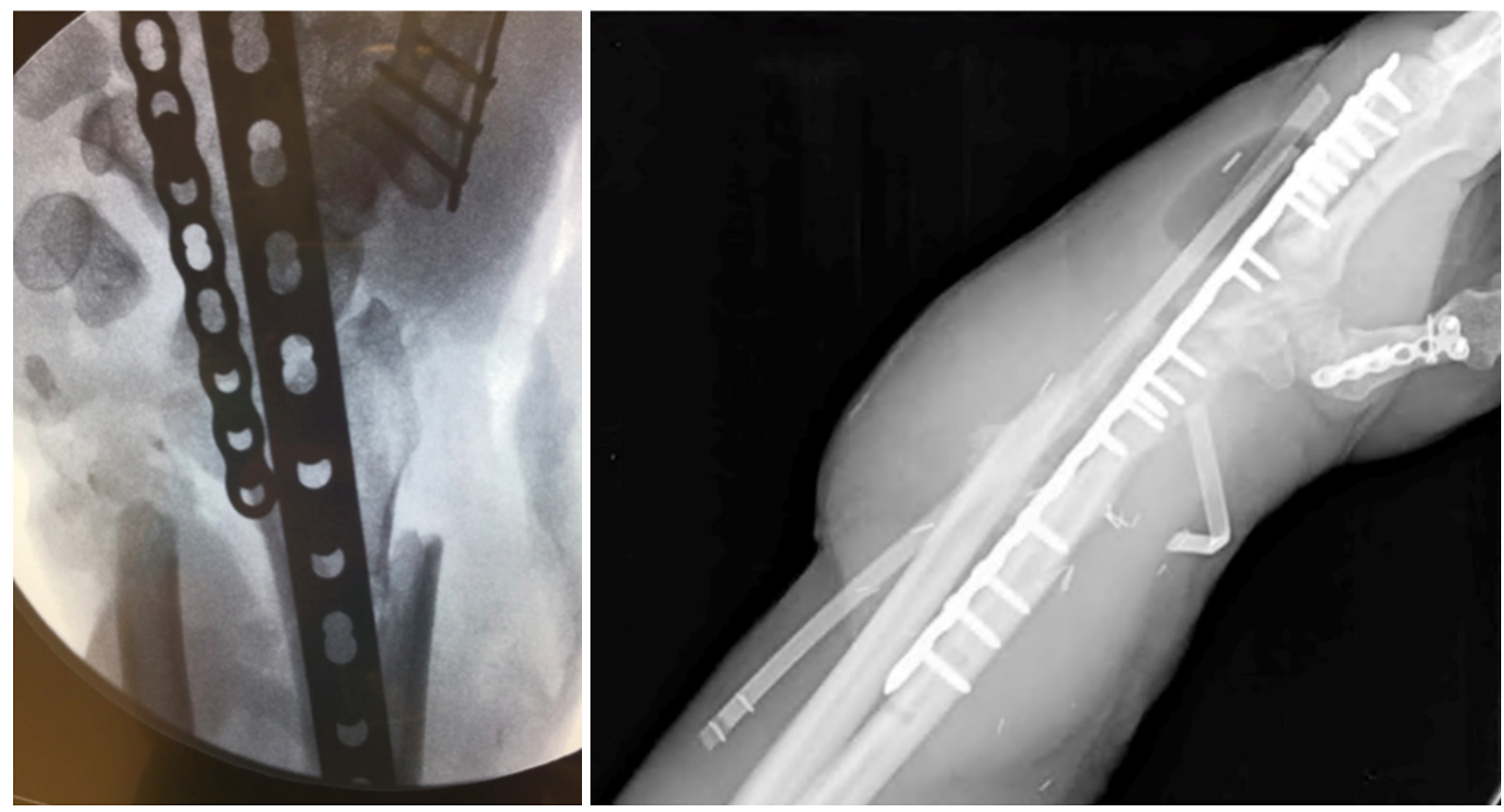

Figure 7. Arthrodesis of carpometacarpal, midcarpal, and radiocarpal joints with dual plate fixation for control of unstable carpal bones and distal radial fracture. Hardware seen on thumb metacarpal was present from previous injury

that was transferred from the right side to the left dorsal wrist and hand and covered with a split thickness skin graft [Figure 8].

At the same time, he underwent first stage extensor tendon reconstruction of the EPL and common extensors to the index through small fingers, using three silicone tendon implants (one secured to the distal 


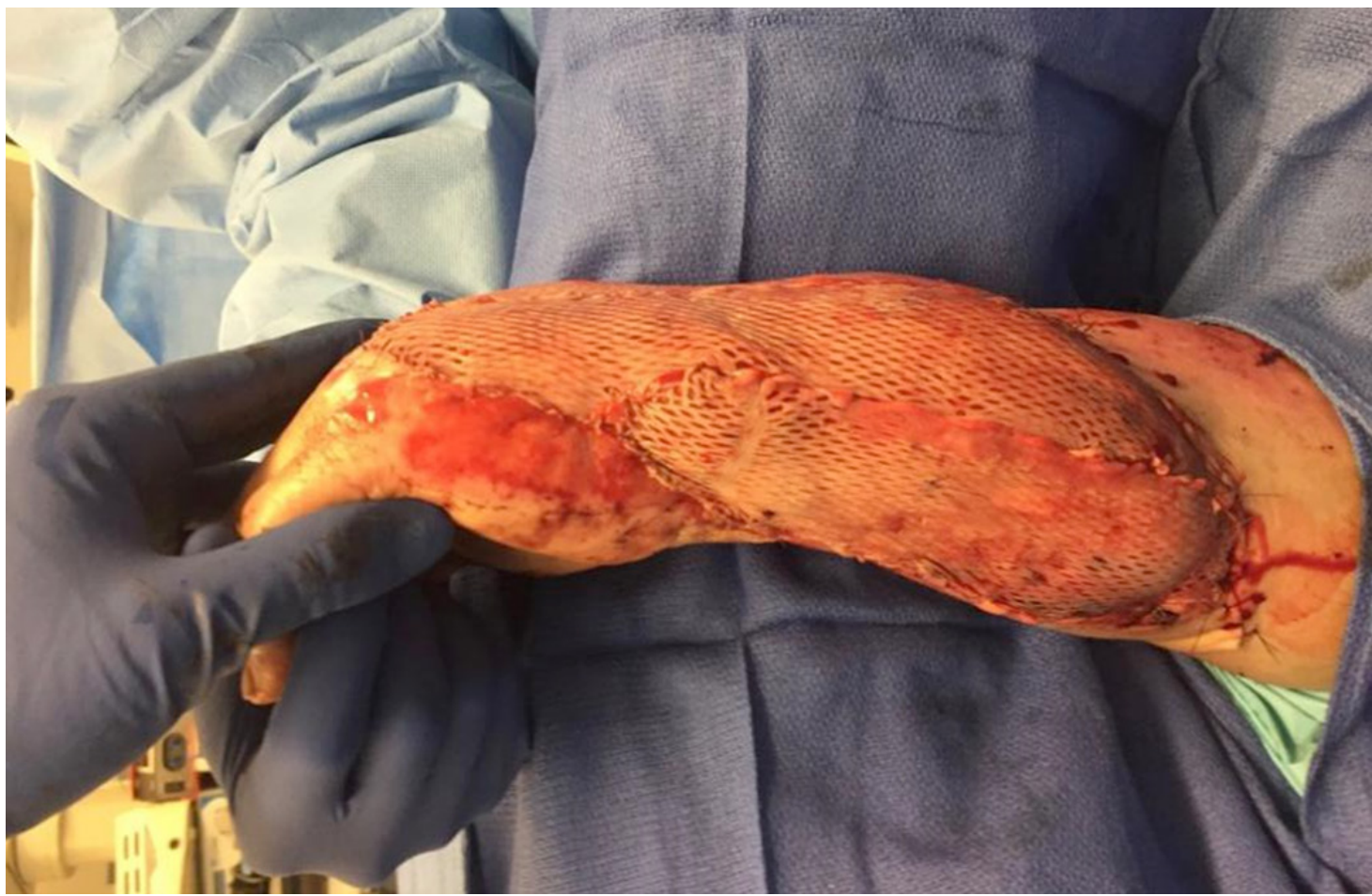

Figure 8. Free latissimus dorsi muscle flap to the defect followed by skin-thickness skin grafting of the muscle belly

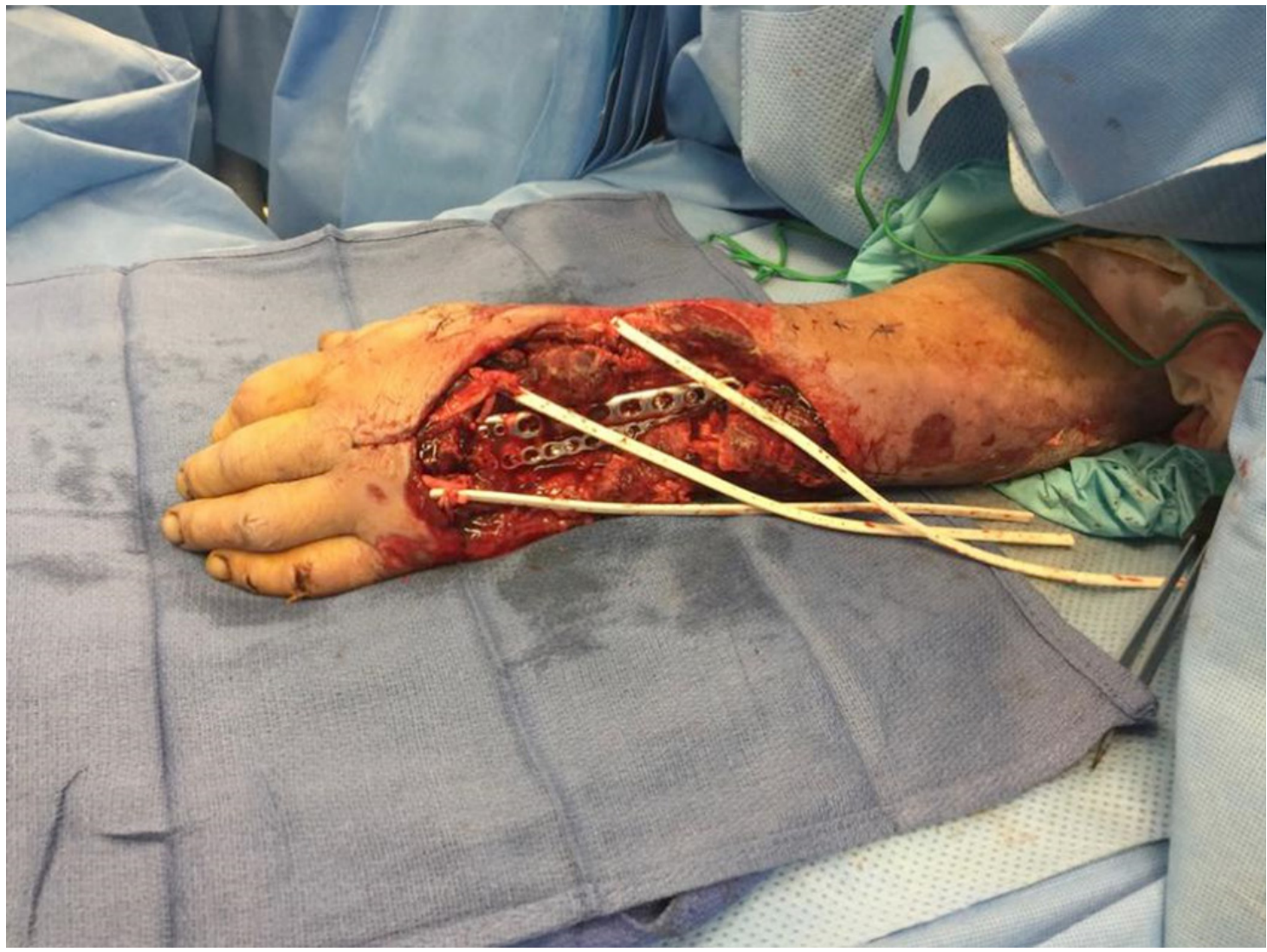

Figure 9. First stage extensor tendon reconstruction with silastic hunter tendon implants (Wright Medical Technology, Memphis, TN) to the extensor hood mechanisms distally 


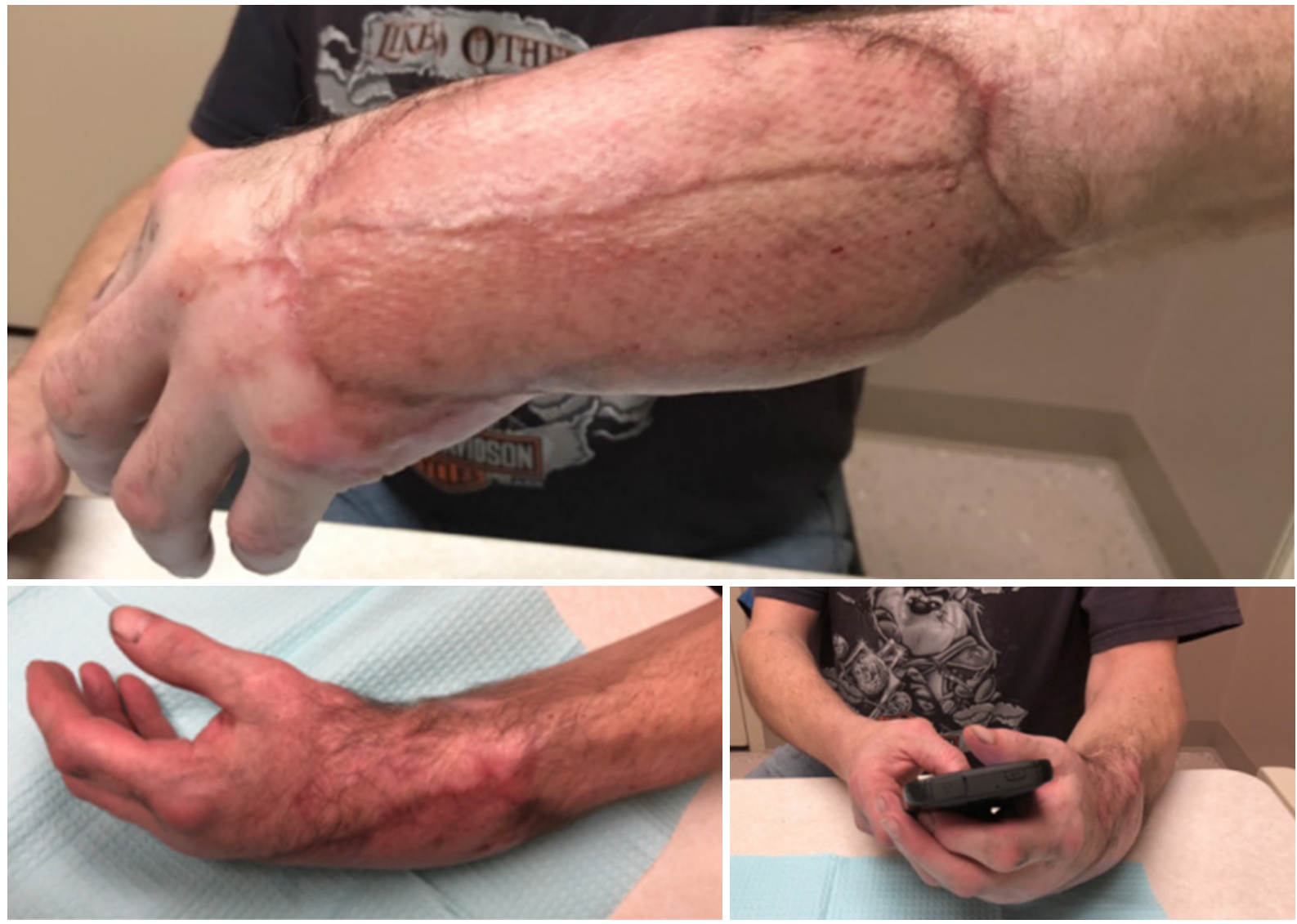

Figure 10. Four months postoperative follow-up showing atrophy of the latissimus muscle with improved contouring and use of the reconstructed hand for activities of daily living

EPL stump, one split to the index and long extensors, and one split to the ring and small extensors) [Figure 9]. These were placed directly on the hardware beneath the muscle flap. The flap contoured nicely to the dorsal of the hand and wrist, and the arthrodesis achieved bony union by three months [Figure 10]. At latest follow up, the second stage extensor tendon reconstruction has been delayed secondary to unplanned incarceration of the patient.

\section{CONCLUSION}

Dorsal shearing injuries to the hand and wrist can be devastating to hand function given their destruction of the soft tissue envelope, extensor tendon, and underlying bony skeleton. Additionally, these injuries pose unique and challenging problems to treating surgeons charged with reconstructing these deficits. Appropriate management begins with stabilization of the traumatic patient followed by thorough and often repeated debridements of the wound. Once ready for reconstruction, goals of reconstruction include bony stabilization, extensor tendon reconstruction, and stable soft tissue coverage. The reconstructive surgeon should assess coverage options based on planned, future surgeries such as staged tendon reconstruction.

Throughout the perioperative period, passive and/or active range of motion modalities are indicated for those joints that are deemed stable and appropriate. This is particularly important if staged debridements or surgeries are required. Extended periods of immobility through multiple stages of surgery can negatively affect eventual outcomes of mobility and function. Once healed, reasonable return of function to a previously mangled hand and wrist can be expected for the patient. 


\section{DECLARATIONS}

\section{Authors' contributions}

Contributed to literature review and writing manuscript: Murthy PG

Contributed surgical cases and photos, contributed to literature review and to writing and editing manuscript: Strohl AB

\section{Availability of data and materials}

Not applicable.

\section{Financial support and sponsorship}

None.

\section{Conflicts of interest}

Both authors declared that there are no conflicts of interest.

\section{Ethical approval and consent to participate}

Not applicable.

\section{Consent for publication}

Not applicable.

\section{Copyright}

(C) The Author(s) 2020.

\section{REFERENCES}

1. Bakri K, Moran SL. Initial assessment and management of complex forearm defects. Hand Clin 2007;23:255-68, vii.

2. Reid DC. Hand injuries requiring skin replacement and restoration of tendon function. Br J Plast Surg 1974;27:5-18.

3. Dautel G. Vascularized toe joint transfers to the hand for PIP or MCP reconstruction. Hand Surg Rehabil 2018;37:329-36.

4. Pappalardo M, Laurence VG, Lin YT. Chimeric free vascularized metatarsophalangeal joint with toe fillet flap: a technique for reconstruction of the posttraumatic metacarpophalangeal joint with concomitant soft tissue defect. J Hand Surg Am 2018;43:193.e1-6.

5. Soutar DS, Tanner NS. The radial forearm flap in the management of soft tissue injuries of the hand. Br J Plast Surg 1984;37:18-26.

6. Mandrekas AD, Zambacos GJ. Reverse-flow radial forearm flap for reconstruction of the hand. Ann Plast Surg 1994;33:536-43.

7. Grobbelaar AO, Harrison DH. The distally based ulnar artery island flap in hand reconstruction. J Hand Surg Br 1997;22:204-11.

8. Zancolli EA, Angrigiani C. Posterior interosseous island forearm flap. J Hand Surg Br 1988;13:130-5.

9. Adani R, Tarallo L, Marcoccio I, Cipriani R, Gelati C, Innocenti M. Hand reconstruction using the thin anterolateral thigh flap. Plast Reconstr Surg 2005;116:467-73; discussion 474-7.

10. Tuncer S, Sezgin B, Sencan A, Sari A. Free serratus fascia flap for reconstruction of soft tissue defects involving the distal upper and lower extremity. Ann Plast Surg 2020;84:672-8.

11. Hirase Y, Kojima T, Bang HH. Double-layered free temporal fascia flap as a two-layered tendon-gliding surface. Plast Reconstr Surg 1991;88:707-12.

12. Logan SE, Alpert BS, Buncke HJ. Free serratus anterior muscle transplantation for hand reconstruction. Br J Plast Surg 1988;41:639-43.

13. Brody GA, Buncke HJ, Alpert BS, Hing DN. Serratus anterior muscle transplantation for treatment of soft tissue defects in the hand. $J$ Hand Surg Am 1990;15:322-7.

14. Kim JT, Kim SK. Hand resurfacing with the superthin latissimus dorsi perforator-based free flap. Plast Reconstr Surg 2003;111:366-70.

15. Kim SW, Lee HJ, Kim JT, Kim YH. Multiple-digit resurfacing using a thin latissimus dorsi perforator flap. J Plast Reconstr Aesthet Surg 2014;67:74-80.

16. Mateev MA, Beermanov KA, Subanova LK, Novikova TV, Shaltakova G. Shape-modified method using the radial forearm perforator flap for reconstruction of soft-tissue defects of the scalp. J Reconstr Microsurg 2005;21:21-4.

17. Ho AM, Chang J. Radial artery perforator flap. J Hand Surg Am 2010;35:308-11.

18. Caroli A, Adani R, Castagnetti C, Pancaldi G, Squarzina PB. Dorsalis pedis flap with vascularized extensor tendons for dorsal hand reconstruction. Plast Reconstr Surg 1993;92:1326-30.

19. Adani R, Marcoccio I, Tarallo L. Flap coverage of dorsum of hand associated with extensor tendons injuries: a completely vascularized single-stage reconstruction. Microsurgery 2003;23:32-9.

20. Cui MY, Shen H. Anterolateral thigh free flap for simultaneous reconstruction of digital extensor tendon and defect of the dorsal hand: a 
case report. Chin J Traumatol 2016;19:309-10.

21. di Summa PG, Sapino G, Cherubino M, et al. Reconstruction of complex soft tissue defects including tendons with anterolateral thigh flap extended to fascia lata: long term recovery and functional outcomes. Microsurgery 2019;39:405-15.

22. Kitazawa T, Shiba M, Tsunekawa K. Free serratus anterior fascial flap combined with vascularized scapular bone for reconstruction of dorsal hand and finger defects. Case Reports Plast Surg Hand Surg 2017;5:1-8.

23. Koshima I. A new classification of free combined or connected tissue transfers: introduction to the concept of bridge, siamese, chimeric, mosaic, and chain-circle flaps. Acta Med Okayama 2001;55:329-32.

24. Scheker LR, Langley SJ, Martin DL, Julliard KN. Primary extensor tendon reconstruction in dorsal hand defects requiring free flaps. $J$ Hand Surg Br 1993;18:568-75.

25. Ozbaydar M, Orman O, Ozel O, Altan E. Multiple extensor tendons reconstruction with hamstring tendon grafts and flap coverage for severe dorsal hand injuries. Hand Surg Rehabil 2017;36:410-5.

26. Hunter J. Artificial tendons. Early development and application. Am J Surg 1965;109:325-38.

27. Schneider LH. Staged flexor tendon reconstruction using the method of Hunter. Clin Orthop Relat Res 1982;171:164-71. 